\title{
An Empirical Model of Occupancy-Queue Relation
}

\author{
Jan Přikryl* Juš Kocijan** \\ * Czech Technical University in Prague, Faculty of Transportation \\ Sciences and Department of Adaptive Systems, UTIA AVCR, Prague \\ (email: prikryl@fd.cvut.cz) \\ ** Jozef Stefan Institute and University of Nova Gorica (email: \\ jus.kocijan@ijs.si)
}

\begin{abstract}
One of the key indicators of traffic control quality in urban traffic control (UTC) systems is the queue length. Even in unsaturated conditions, longer queues indicate longer travel delays and higher fuel consumption. With the exception of some expensive surveillance equipment, the queue length itself cannot be measured directly. Many methods that estimate the queue length from detector measurements are used in engineering practice, ranging from simple to elaborate ones. The proposed method is based on Gaussian process (GP) model of the occupancy-queue relationship. It can handle data uncertainties and provides more information about the quality of the queue length prediction.
\end{abstract}

Keywords: Queue estimation, Traffic control, Uncertainty

\section{INTRODUCTION}

Queue length has been regarded as one of the key parameters in the process of signal plan design, as estimates of queue length may be used as a part of a criterion that is minimised by urban traffic control (UTC) systems that provide coordinated control of signalised intersections. Numerous studies discuss the problem of queue development (Friedrich et al., 2003; Mystkowski and Khan, 1998; Viloria et al., 2000; van Zuylen and Viti, 2006). Typical queueing models are for example those of Akcelik (1980), Transportation Research Board (2000), Hensher and Button (2000), or Mück (2002). These models are derived from underlying physical principles of the queue formation and dissipation processes and include some adhoc corrections accounting for the stochastic nature of the queuing process.

Stochastic properties of queue development are directly taken into account by Markov chain models (van Zuylen and Viti, 2003, 2006). This class of models describes queueing as a stochastic process with probabilities of queue change being given by probability distributions.

The third class of models found in literature are black-box models trying to predict the queue length based on known "training" data. These include autoregressive models (Ho and Hwang, 1994), neural networks (Chang and Su, 1995; Ledoux, 1997), combination of neural networks and fuzzy logic (Quek et al., 2006), or neural network constructed with the help of genetic algorithms (Vlahogianni et al., 2005).

All the models mentioned above compute queue length from vehicle count provided by upstream detectors. With the exception of Diakaki (1999) and recent publications of Papageorgiou and Vigos (2008) and Vigos et al. (2008) (which concentrate on estimating the total number of vehicles), detector time-occupancy is not used to provide additional information about the queue formation process, although this quantity is usually provided together with the vehicle count by an intersection controller.

The reason for disregarding time-occupancy information can be twofold: First, pure time-occupancy gives us a reasonable measure of queue length only for limited range of queue tails. If the distance from the detector to the downstream queue tail is high, the time-occupancy stays low regardless of the queue length. If the queue tail reaches upstream far behind the detector, the time-occupancy will be high regardless of the queue length. Second, the readings from the detector are strongly influenced by other parameters of the traffic flow, as inter-vehicle gaps, vehicle speeds, and their length.

Recently, several papers appeared that try to make use of the time-occupancy measurements for providing additional information about the traffic state at an approach to a signalised intersection. Chang et al. (2000) combine vehicle count and time-occupancy measurements with known signal state and a model of vehicle dynamics to estimate the queue length. Another approach has been taken by Papageorgiou and Vigos (2008) who start with thorough theoretical analysis of the time-occupancy measurements and follow with a Kalman filter implementation of vehicle count estimator (Vigos et al., 2008).

In this paper we present an empirical approach to queue length estimation from time-occupancy data, that is meant as augmentation of the existing methods and that is able to provide estimation of queue length in the vicinity of a loop detector based on sparse occupancy measurements.

The paper is further organised as follows: in Section 2 we will briefly discuss the practical observations that 
led to the method proposed in this article. Section 3 contains a short introduction to Gaussian process (GP) models that will be used as a base of the queue length estimation method. This Section also lists the parameters of the GP model used in the paper. Results of the queue length estimation using the model and their discussion are presented in Section 4.

\section{MOTIVATION}

In the last few years the research group at UTIA developed first approximation of a state-space queue length model for urban arteries based on ideas of Homolová and Nagy (2005). This model is based on vehicle conservation law and non-linear Kalman filtering, and uses solely information from strategic upstream detectors.

When adopting this model to a smaller network equipped also with dilemma-zone detectors, the measurements provided by these detectors will be ignored by the model, although they could reasonably improve the queue length estimate. Hence, we would like to find a mechanism for a dilemma-zone detector to provide additional information about the queue tail development, and to identify situations where the detector will not contribute to a reasonable estimate due to saturation. In order to suppress the zero speed-zero occupancy phenomenon observed by Papageorgiou and Vigos (2008), we suppose that the sizes of the detector loops exceed the typical maximum gap between vehicles.

We have several limiting factors, though:

- the placement of the detectors in the network is fixed,

- additional detectors are not an option,

- due to equipment limitations we have relatively sparse measurements with period of 90 seconds.

Given the nature of time-occupancy measurements, the position of the queue tail will have different influence on detectors placed in different distances from the stop-bar. It is clear that the further is the detector installed from the stop-bar, the less occupancy changes caused by short queues on occupancy can be measured. On the other hand, if the queue tail reaches behind the detector, the queue length cannot be measured anymore. As we can see from a simulated occupancy-queue length graph in Figure 1, the position of the detector alone causes vertical shifts in the graphs. We will therefore identify the model for a fixed detector distance from the stop-bar.

However, as we can also see in Figure 1, if we take into account variable lengths of the green signal as a result of vehicle actuated control mechanisms, the resulting measurements become quite noisy as the maximum queue length is definitely influenced by the length of the downstream green signal, and the readings of the detector are influence by the amount of free flow of vehicles during the green phase.

We are therefore also logging the changes in signal group states at the intersection controller and we would like to build a model that makes use of green length and occupancy measurements to estimate the number of queueing vehicles.

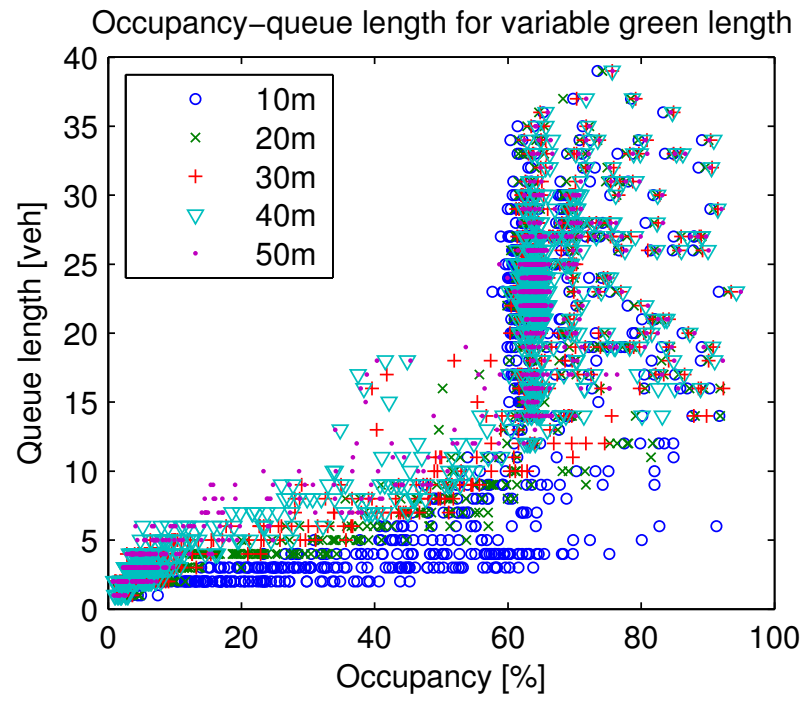

Fig. 1. Occupancy-queue length graph for different detector distances from the stop-bar. Note the strong noise in the observed data that is due to changes in the length of green signal. Simulated in Aimsun (Transport Simulation Systems, 2008).

Our queue model is inspired by Diakaki (1999) and it is based on the experiments with existing queue length estimation model of Homolová and Nagy (2005). This model does not behave well for high-occupancy situations and provides a point estimate only. While it uses a linear dynamic first-order formula for queue-occupancy relationship, our initial experiments indicated that the additional information provided by the dynamic part of the model does not bring significant improvements to the results obtained and the model can be expressed as static. We could use other models that already exist, but we would like to have interval estimates of queue length rather than point estimates. This is the reason why we chose the Gaussian process model.

\section{GAUSSIAN PROCESS MODELS}

The Gaussian process model is an example of the use of a flexible, probabilistic, non-parametric model with uncertainty predictions. It fits naturally in the Bayesian modelling framework in which instead of parameterising mapping function $f(\mathbf{x})$, a prior is placed directly on the space of possible functions $f(\mathbf{x})$ which could represent the nonlinear mapping from input vector $\mathbf{x}$ to output $y$. Its use and properties for modelling are reviewed in Rasmussen and Williams (2006). In our particular case the input vector $\mathbf{x}$ will be composed of time-occupancy and green length and the output $y$ will correspond to the estimated queue length.

A Gaussian process is a generalisation of the Gaussian probability distribution. It can be viewed as a collection of random variables $f\left(\mathbf{x}_{i}\right)$ with joint multivariate Gaussian distribution: $f\left(\mathbf{x}_{1}\right), \ldots, f\left(\mathbf{x}_{n}\right) \approx \mathcal{N}(0, \boldsymbol{\Sigma})$, where $\mathcal{N}(\cdot, \cdot)$ stands for Gaussian distribution determined with mean value and variance, where covariance matrix element $\Sigma_{p q}=\operatorname{Cov}\left(y_{p}, y_{q}\right)=C\left(\mathbf{x}_{p}, \mathbf{x}_{q}\right)$ gives the covariance between values of the functions $y_{p}=f\left(\mathbf{x}_{p}\right)$ and $y_{q}=f\left(\mathbf{x}_{p}\right)$. Thus, the mean $\mu(\mathbf{x})$ (usually assumed to be zero) and the 
covariance function $C\left(\mathbf{x}_{p}, \mathbf{x}_{q}\right)$ fully specify the Gaussian process. Note that the covariance function $C(\cdot, \cdot)$ can be any function having the property of generating a positive definite covariance matrix.

A common choice is

$$
C\left(\mathbf{x}_{p}, \mathbf{x}_{q}\right)=v_{1} \exp \left[-\frac{1}{2} \sum_{d=1}^{D} w_{d}\left(x_{p}^{d}-x_{q}^{d}\right)^{2}\right]+\delta_{p q} v_{0},
$$

where $\boldsymbol{\Theta}=\left[w_{1}, \ldots, w_{D}, v_{1}, v_{0}\right]^{T}$ are the 'hyperparameters' of the covariance functions, $v_{0}$ is estimated noise variance, $v_{1}$ is the estimate of the vertical scale of variation, $D$ is the input dimension, $x_{p}^{d}$ and $x_{q}^{d}$ are $d^{\text {th }}$ components of input vectors $\mathbf{x}_{p}, \mathbf{x}_{q}$ and $\delta_{p q}=1$ if $p=q$ and 0 otherwise. The covariance function (1) is composed of two parts: the Gaussian covariance function for the modelling of system function and the covariance function for the modelling of noise. The noise, in our case, is presumed to be white. Other forms of covariance functions suitable for different applications can be found in Rasmussen and Williams (2006).

For a given problem, the parameters in $\Theta$ are learned (identified) using the data at hand. After the learning, one can use the $w$ parameters as indicators of 'how important' the corresponding input components (dimensions) are: if $w_{d}$ is zero or near zero it means that the inputs in dimension $d$ contain little information and could possibly be removed.

Consider a set of $N D$-dimensional input vectors $\mathbf{X}=$ $\left[\mathbf{x}_{1}, \mathbf{x}_{2}, \ldots, \mathbf{x}_{N}\right]$ and a vector of output data $\mathbf{y}=\left[y_{1}, y_{2}, \ldots, y_{N}\right]^{T}$. Based on the data $(\mathbf{X}, \mathbf{y})$, and given a new input vector $\mathbf{x}^{*}$, we wish to find the predictive distribution of the corresponding output $y^{*}$. Unlike other models, there is no model parameter determination as such, within a fixed model structure. With this model, most of the effort consists in tuning the parameters of the covariance function. This is done by maximisation of the log-likelihood

$$
\begin{aligned}
\mathcal{L}(\boldsymbol{\Theta}) & =\log (p(\mathbf{y} \mid \mathbf{X})) \\
& =-\frac{1}{2} \log (|\mathbf{K}|)-\frac{1}{2} \mathbf{y}^{T} \mathbf{K}^{-1} \mathbf{y}-\frac{N}{2} \log (2 \pi)
\end{aligned}
$$

where $\boldsymbol{\Theta}$ is the vector of hyperparameters and $\mathbf{K}$ is the $N \times N$ training covariance matrix. The calculation of the log-likelihood and its derivatives due to the optimisation algorithm involves the computation of the inverse of the $N \times N$ covariance matrix $\mathbf{K}$ at every iteration, which can become computationally demanding for large $N$. Nevertheless, the number of parameters to be optimised is small $(D+2$, see Equation (1)), which means that optimisation convergence might be faster and that the 'curse of dimensionality' so common to black-box identification methods is circumvented or at least decreased.

The described approach can be easily utilised for regression calculation. Based on training set $\mathbf{X}$ a covariance matrix K of size $N \times N$ is determined. As already mentioned, the aim is to find the distribution of the corresponding output $y^{*}$ at some new input vector $\mathbf{x}^{*}=\left[x_{1}(N+1), x_{2}(N+\right.$ $\left.1), \ldots, x_{D}(N+1)\right]^{T}$.

For a new test input $\mathrm{x}^{*}$, the predictive distribution of the corresponding output $y^{*}$, over cases in the training set $(\mathbf{X}, \mathbf{y})$ is $y^{*} \mid(\mathbf{X}, \mathbf{y}), \mathbf{x}^{*}$ and is Gaussian, with mean and variance

$$
\begin{aligned}
\mu\left(\mathbf{x}^{*}\right) & =\mathbf{k}\left(\mathbf{x}^{*}\right)^{T} \mathbf{K}^{-1} \mathbf{y}, \\
\sigma^{2}\left(\mathbf{x}^{*}\right) & =\kappa\left(\mathbf{x}^{*}\right)-\mathbf{k}\left(\mathbf{x}^{*}\right)^{T} \mathbf{K}^{-1} \mathbf{k}\left(\mathbf{x}^{*}\right),
\end{aligned}
$$

where $\mathbf{k}\left(\mathbf{x}^{*}\right)=\left[C\left(\mathbf{x}^{1}, \mathbf{x}^{*}\right), \ldots, C\left(\mathbf{x}^{N}, \mathbf{x}^{*}\right)\right]^{T}$ is the $N \times 1$ vector of covariances between the test and training cases, and $\kappa\left(\mathbf{x}^{*}\right)=C\left(\mathbf{x}^{*}, \mathbf{x}^{*}\right)$ is the covariance between the test input and itself. Vector $\mathbf{k}\left(\mathbf{x}^{*}\right)^{T} \mathbf{K}^{-1}$ in (3) can be interpreted as a vector of smoothing terms which weights the training outputs from $\mathbf{y}$ to make a prediction at the test point $\mathrm{x}^{*}$.

In our particular case $\mathbf{x}_{i}=\left[O_{i}, z_{i}\right]$, where $O_{i}$ is the occupancy and $z_{i}=g_{i} / T_{\mathrm{c}}$ is the relative green length computed as a ratio of the real green length $g_{i}$ and the cycle length $T_{\mathrm{c}}$. The corresponding component of the output vector $\mathbf{y}$ is the queue length measured for the given $O_{i}$ and $z_{i}$.

The reasons to select modelling with Gaussian process models are small amounts of data relative to the number of selected regressors, data corrupted with noise and measurement errors and the need for the measure of model prediction confidence. If there is not enough data or it is heavily corrupted with noise, even the Gaussian process model cannot perform well, but in that case the inadequacy of the model and the identification data is indicated through higher variance of the predictions.

The utility to provide the information about the model confidence made Gaussian process models attractive for modelling case studies in various domains like: chemical engineering Kocijan and Likar (2007) and process control Likar and Kocijan (2007), biomedical engineering Faul et al. (2007), biological systems Ažman and Kocijan (2007), environmental systems Grašič et al. (2006), power systems Leith et al. (2004) and engineering Leithead et al. (2005), motion recognition Wang et al. (2008), etc., to list just a few. We believe that this utility makes it interesting also for use in the domain of traffic modelling and its applications.

We should note that there is no assumption that signals measured in a traffic process will have Gaussian distribution. The Gaussian process prior is put over the space of functions meaning that every prediction that is made by GP model has Gaussian distribution. This implies that every prediction that is made has some most likely value and the less likely values are equally possible on both sides of the most likely value. This is, by our convenience, not unrealistic.

\section{RESULTS}

As it is not possible for us to conduct deliberate experiments in real-world traffic, in order to demonstrate the behaviour of the proposed GP model, one day of traffic was simulated on a simple network (see Figure 2) using the Aimsun micro-simulator (Transport Simulation Systems, 2008) that, once calibrated, should be able to closely mimic the real traffic (Fang and Elefteriadou, 2005). The results shown in this section were obtained using data from detector placed at distance of 20 metres (approximately 56 vehicles) from the stop-bar. This distance was selected as an average distance of dilemma-zone detectors at our test 


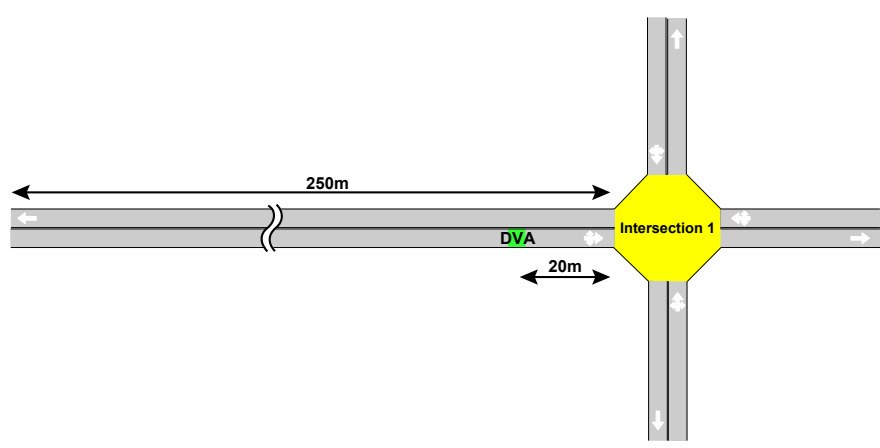

Fig. 2. Test network model. The detector DVA is used for measurements.

site. The traffic demand data used the simulation were real-world measurements from our test site at PragueZličín. In order to mimic the behaviour of the vehicle actuated traffic signal control, the duration of the green signal varied from 16 to 55 seconds in a 90 second cycle. Every 90 seconds of the simulation the maximum queue length, the relative green length, and detector measurements were stored, resulting in 960 samples of data consisting of green length, occupancy, and maximum queue length. This approach corresponds to the common traffic engineering practice in Prague where measurements are collected every 90 seconds.

The 960 simulated measurements were then divided into two groups of 254 training and 706 validation data samples using stratified sampling of a $2 \mathrm{D}$ histogram of queue lengths versus measured occupancy. The size of a queue length bin was 2 vehicles, the size of an occupancy bin was $4 \%$. As the process of GP identification (2) requires a repetitive inverse of the training covariance matrix, the number of 254 training samples was selected by hand as a limit providing the tuned covariance function in an acceptable time on our hardware.

When we first identified a GP model for the whole training data set, the high variance of the measurements in the high occupancy region dominated the whole prediction. Situation like this may happen due to selection of the covariance function in the GP identification process (Rasmussen and Williams, 2006). To obtain lower variance of queue length estimation in the low occupancy region, we opted for a hybrid model, dividing the modelled occupancy into two regions with different operating regimes. The position of the split is data dependent and is given by the abrupt change in variance observed in the measurement data and in our case can be taken at $O=50 \%$. The resulting composite model is shown in Figure 3. In all figures, the relative green length component of the model has been omitted for clarity reasons.

Figure 4 shows the performance of the model for its own training data in low variance region, Figure 5 contains queue estimation for the same region using the validation data set. Looking at the confidence interval for queue length we can observe that if the maximum queue length estimates are close to zero, the confidence interval predicted by the GP model may contain negative values. This is the nature of the estimation process as GP models can not use truncated Gaussian distributions and in such a case the confidence interval lower bound can be clamped

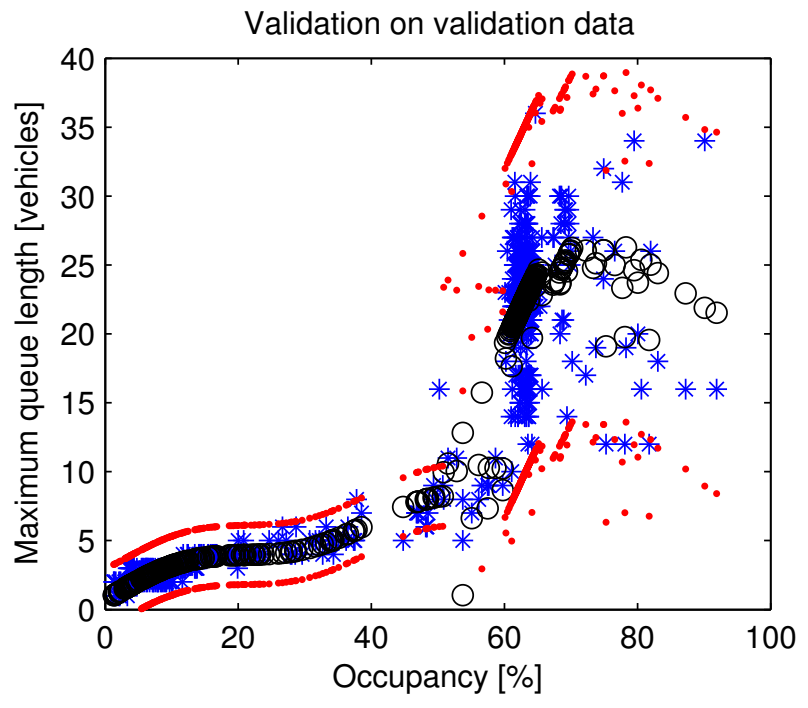

Fig. 3. Two components of the model merged together on validation data. Stars denote the correct data, circles denote the predicted mean value, and red/gray dots limit the $95 \%$ confidence interval.

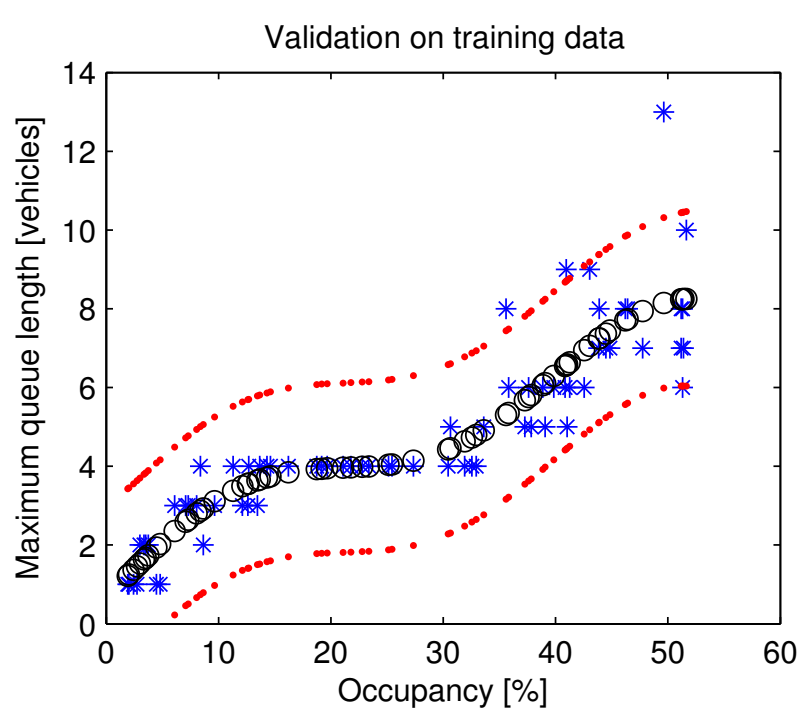

Fig. 4. Validation on training data of the low noise component of the model. Stars denote the correct data, circles denote the predicted mean value, and red/gray dots limit the $95 \%$ confidence interval.

at zero. This clamping corresponds in fact to adding a prior knowledge "queue length can not be negative" to the model.

The behaviour of the model in the high variance region is shown in Figure 6 for the training data and in Figure 7 for the validation data set. We can see that in this case the observed variance in the training data (due to detector saturation and uncertainty in measurements, where certain occupancy value may correspond to different queue lengths) results also in predictions with high variance. This is the correct result as the quality of measured data does not permit better queue length prediction. 


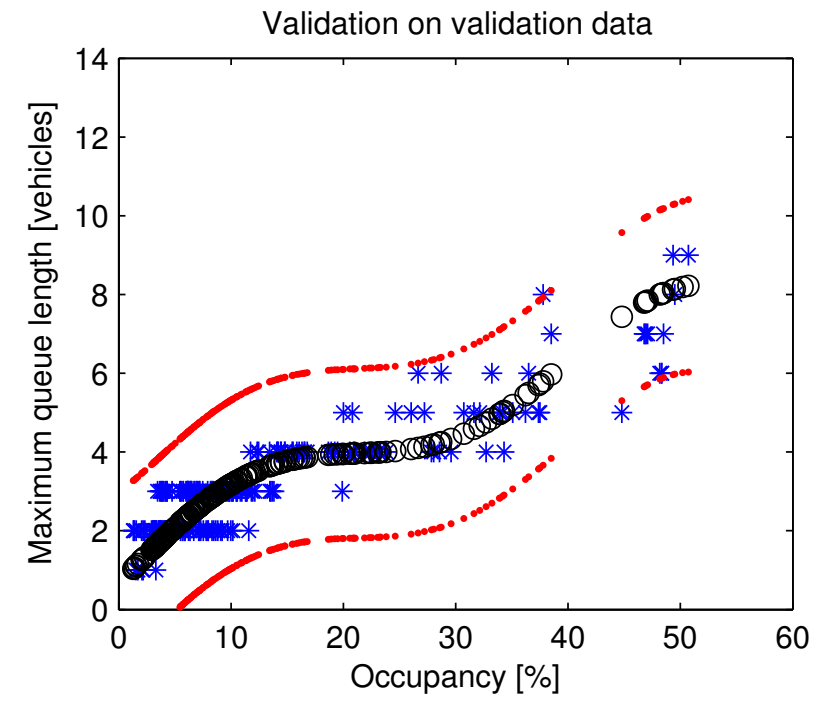

Fig. 5. Validation on validation data of the low noise component of the model. Stars denote the correct data, circles denote the predicted mean value, and $\mathrm{red} /$ gray dots limit the $95 \%$ confidence interval.

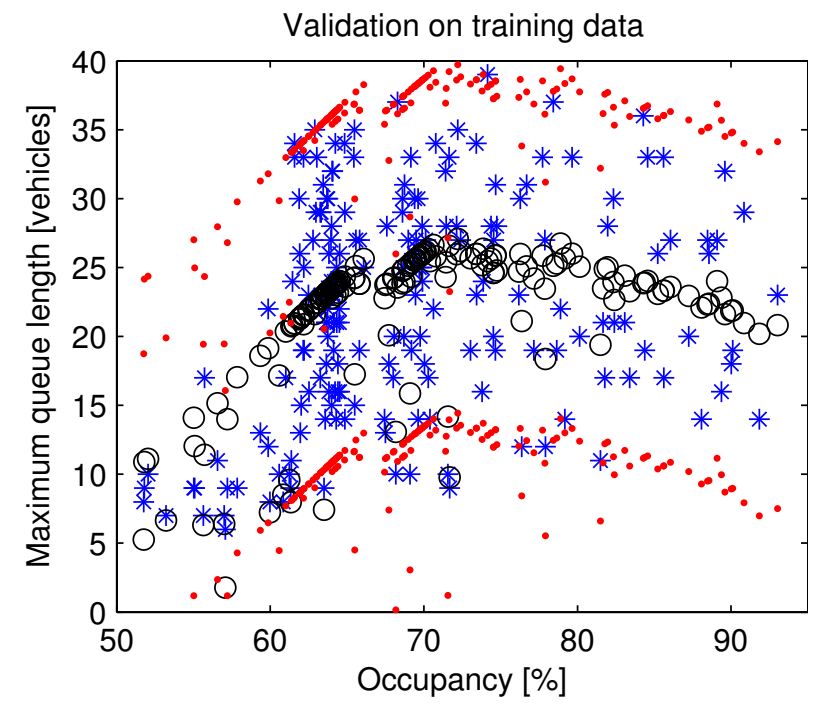

Fig. 6. Validation on training data of the high noise component of the model. Stars denote the correct data, circles denote the predicted mean value, and $\mathrm{red} /$ gray dots limit the $95 \%$ confidence interval.

\section{CONCLUSIONS}

We have presented an empirical static model for determining queue length from sparse occupancy data and green signal length measurements, based on Gaussian process framework. Due to the nature of the underlying identification algorithm of the Gaussian process, the presented version of the model is hybrid, consisting of two different models for low-saturation and high-saturation conditions. The presented model is based on simulated data as we do not have access to equipment that would allow us to measure the queue directly.

Experimental results confirm that occupancy measurements may provide reasonable queue length estimates for unsaturated conditions on the detector and that the GP-

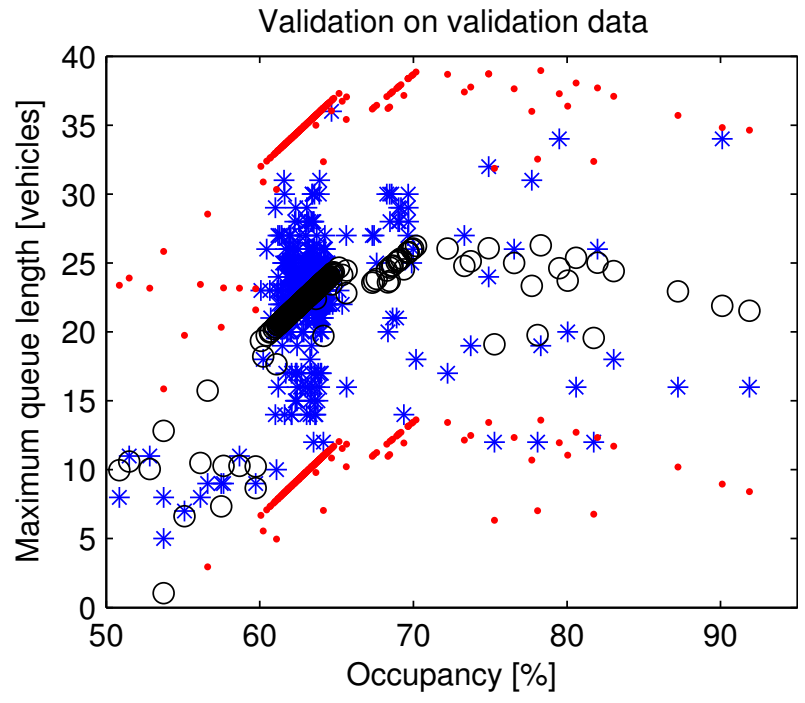

Fig. 7. Validation on validation data of the high noise component of the model. Stars denote the correct data, circles denote the predicted mean value, and red/gray dots limit the $95 \%$ confidence interval.

based model can identify situations where the occupancy measurements will not provide reasonable estimate due to detector saturation. The effect of rising queue length on standard deviation of its estimate is similar to the observations of van Zuylen and Viti (2003) for an intensitybased model. As our model is meant to augment existing intensity-based estimator, we will in the latter case ignore the information provided by the GP model, although we may use the knowledge e.g. to set some lower bound for our intensity-based queue length estimator.

The main advantage of using a GP model over different modelling approaches is that it tells what is the confidence of the model predictions, which is valuable additional information - results provided by different models may or may not verify well in practice, but the measure of confidence in our model reflects the uncertainty of the predictions due to, for example, lack of representative data, or due to uncertainty in the original training data set. Similar to Gaussian process models, methods based on linear regression can also provide an estimate of uncertainty (or confidence in prediction), but this uncertainty would be a constant in entire modelled region. In our case it is not, though it does not vary much.

Many possible extensions to this model can be developed: The hybrid nature of the model with a split between the high- and low-variance regions can be eliminated by using a different formulation of the covariance function (1). Such a model would then be able to adapt to inhomogeneous variances in the input data. Unfortunately, development of such an approach is not straightforward. Also, the model is static, meaning that it does not make use of information about traffic dynamics, as some of the intensity based approaches do (Diakaki, 1999). If a higher sampling rate could be achieved (for example, 5 or 10 seconds instead of current 90 seconds) the dynamic behaviour of the queueing process could be possibly captured by a dynamic model. Such a model would provide better estimates and improve the reliability of the estimates in the region of detector 
saturation. Finally, some of the ideas provided by $\mathrm{Pa}$ pageorgiou and Vigos (2008) may be used to combine the sparse time-occupancy measurements with other information about the traffic on an approach to a signalised intersection.

\section{ACKNOWLEDGEMENTS}

The authors would like to thank anonymous reviewers for their valuable comments and suggestions. This work has been supported in part by the Ministry of Education, Youth and Sports of the Czech Republic project 1M0572 (research centre "Data-Algorithms-Decision Making") and by the bilateral Czech-Slovene project BI-CZ/07-08-11.

\section{REFERENCES}

Akcelik, R. (1980). Time-dependent expressions for delay, stop rate and queue length at traffic signals. Technical Report AIR 367-1, Australian Road Research Board.

Ažman, K. and Kocijan, J. (2007). Application of gaussian processes for black-box modelling of biosystems. ISA Transactions, 46(4), 443-457.

Chang, G.L. and Su, C.C. (1995). Predicting intersection queue with neural network models. Transportation Research Part C, 3(3), 175-191.

Chang, J., Liebermann, E.B., and Shenk Prassas, E. (2000). Queue estimation algorithm for real-time control policy using detector data. Technical report, KLD Associates.

Diakaki, C. (1999). Integrated Control of Traffic Flow in Corridor Networks. Ph.D. thesis, Technical University of Crete, Department of Production Engineering and Management.

Fang, F.C. and Elefteriadou, L. (2005). Some guidelines for selecting microsimulation models for interchange traffic operational analysis. Journal of Transportation Engineering, 131(7), 535-543.

Faul, S., Gregorčič, G., Boylan, G., Marnane, W., Lightbody, G., and Connolly, S. (2007). Gaussian process modelling of eeg for the detection of neonatal seizure. IEEE Transactions on Biomedical Engineering, 54(12), 2151-2162.

Friedrich, B., Matschke, I., Almasri, E., and Mück, J. (2003). Data fusion techniques fo adaptive traffic signal control. In Proceedings of 2003 IFAC Control in Transportaion Systems Conference.

Grašič, B., Mlakar, P., and Božnar, M.Z. (2006). Ozone prediction based on neural networks and gaussian processes. Nuovo Cimento della Societa Italiana di Fisica, Sect. C, 29(6), 651-662.

Hensher, D.A. and Button, K.J. (eds.) (2000). Handbook of Transport Modelling. Pergamon Press.

Ho, C.H. and Hwang, T.L. (1994). Modeling real-time dynamic queue length for urban traffic control systems. In Proceedings of the Intelligent Vehicles '94 Symposium, 438-442.

Homolová, J. and Nagy, I. (2005). Traffic model of a microregion. In P. Horáček, M. Šimandl, and P. Zítek (eds.), Preprints of the 16th World Congress of the International Federation of Automatic Control, 1-6. IFAC, Prague.

Kocijan, J. and Likar, B. (2007). Gas-liquid separator modelling and simulation with gaussian process mod- els. In Proceedings of the 6th EUROSIM Congress on Modelling and Simulation - EUROSIM 2007, 7 pages. Ljubljana.

Ledoux, C. (1997). An urban traffic flow model integrating neural networks. Transportation Research Part C, 5(5), $287-300$.

Leith, D.J., Heidl, M., and Ringwood, J. (2004). Gaussian process prior models for electrical load forecasting. In Proceedings of 2004 International Conference on Probabilistic Methods Applied to Power Systems, 112-117. IEEE.

Leithead, W.E., Zhang, Y., and Neo, K.S. (2005). Wind turbine rotor acceleration: Identification using gaussian regression. In Proceedings of International conference on informatics in control automation and robotics (ICINCO), 84-91. Barcelona.

Likar, B. and Kocijan, J. (2007). Predictive control of a gas-liquid separation plant based on a gaussian process model. Computers and Chemical Engineering, 31(3), $142-152$.

Mück, J. (2002). Estimation methods for the state of traffic at traffic signals using detectors near the stopline. Traffic Engineering and Control, 43(11), 429.

Mystkowski, C. and Khan, S. (1998). Estimating queue lengths using SIGNAL94, SYNCHRO3, TRANSYT-7F, PASSER II-90, and CORSIM. In Proceedings of 78th Transportation Research Board Annual Meeting.

Papageorgiou, M. and Vigos, G. (2008). Relating timeoccupancy to space-occupancy and link vehicle-count. Transportation Research Part C, 16, 1-17.

Quek, C., Pasquier, M., and Lim, B.B.S. (2006). Poptraffic: A novel fuzzy neural approach to road traffic analysis and prediction. IEEE Transactions on Intelligent Transportation Systems, 7(2), 133-146.

Rasmussen, C.E. and Williams, C.K. (2006). Gaussian Processes for Machine Learning. MIT Press, Cambridge, MA.

Transport Simulation Systems (2008). Aimsun: Advanced interactive microscopic simulator for urban and nonurban networks. URL http://www. aimsun. com.

Transportation Research Board (2000). Highway Capacity Manual 2000. Transportation Research Board.

van Zuylen, H.J. and Viti, F. (2006). Delay at controlled intersections: the old theory revised. In Proceedigns of the 2006 IEEE Intelligent Treansportation Systems Conference, 68-73.

van Zuylen, H.J. and Viti, F. (2003). Uncertainty and the dynamics of queues at controlled intersections. In Proceedings of 2003 IFAC Control of Transporation Systems Conference.

Vigos, G., Papageorgiou, M., and Wang, Y. (2008). Realtime estimation of vehicle-count within signalized links. Transportation Research Part C, 16, 18-35.

Viloria, F., Courage, K., and Avery, D. (2000). Comparison of queue-length models at signalized intersections. Transportation Research Record, 1710, 222-230.

Vlahogianni, E.I., Karlaftis, M.G., and Golias, J.C. (2005). Optimized and meta-optimized neural networks for short-term traffic flow prediction: A genetic approach. Transportation Research Part C, 13, 211-234.

Wang, J.M., Fleet, D.J., and Hertzmann, A. (2008). Gaussian process dynamical models for human motion. IEEE Transactions on Pattern Analysis and Machine Intelligence, 30(2), 283-298. 